\title{
Análisis espacio-temporal del incumplimiento de normas legales sobre el confinamiento en Colombia por COVID-I 9
}

\section{Spatial and temporal analysis of the breach of legal regulations regarding the Colombian COVID-I 9 lockdown}

\section{Análise espaço-temporal da infringência de normas legais sobre o confinamento na Colômbia pelo COVID-I 9}

\author{
José Ignacio Ruiz-Pérez ${ }^{\mathrm{a} *}$ | Juan Aparicio Barrera ${ }^{\mathrm{b}}$ \\ a https://orcid.org/0000-0002-8996-9736 Universidad Nacional de Colombia, Bogotá, Colombia \\ ${ }^{\text {b }}$ https://orcid.org/0000-000 I-5620-I 766 Policía Nacional de Colombia, Bogotá, Colombia
}

- Fecha de recepción: 2020-05-24

- Fecha concepto de evaluación: 2020-07-03

- Fecha de aprobación: 2020-07-07 https://doi.org//0.22335/rlct.v/2i3.125 I

\begin{abstract}
Para citar este artículo / To reference this article / Para citar este artigo: Ruiz-Pérez, J.I., \& Aparicio,J. (2020).Análisis espacio-temporal del incumplimiento de normas legales sobre el confinamiento en Colombia por COVID-19. Revista Logos Ciencia \& Tecnología, I2(3), 20-32. https://doi.org/I0.22335/rlct.vI2i3.I25I
\end{abstract}

\section{RESUMEN}

El objetivo de este trabajo fue identificar los patrones espaciales y temporales de siete comportamientos contrarios a la convivencia (CCC) durante las medidas de confinamiento aplicadas a nivel nacional en Colombia durante 47 días. Para esto se analizaron 363439 registros administrativos de CCC recolectados por la Policía Nacional desde el 16 de marzo al I de mayo del 2020, con la siguiente información: hora, día, fecha, tipo de comportamiento, edad y departamento del infractor. Los resultados mostraron: (a) relaciones temporales entre el número de casos diarios o acumulados de COVID-19 y las tasas de CCC por 100000 habitantes, (b) concentración de los CCC entre las 8: 00 a. m. y las 12:00 p. m. 0 entre las 2:00 p. m. y las 6 p. m., (c) mayor frecuencia de consumo de bebidas alcohólicas los fines de semana, (d) con relación a la edad, la franja de 18 a 24 años es la que presenta mayor comisión de medidas correctivas.

Palabras clave: COVID-19, epidemia, confinamiento, salud pública, policía 


\section{ABSTRACT}

The objective of this study was to identify the spatial and temporal patterns of seven behaviours contrary to coexistence $(B C C)$ during the lockdown measures applied at a national level in Colombia for 47 days. For this, 363,439 administrative records collected by the National Police from March 16 to May I, 2020 regarding BCCs were analysed, all including the following information: time, day, date, type of behaviour, age and state or department of the offender. The results showed: (a) temporal relationships between the number of daily or accumulated cases of COVID-19 and the rates of BCCs per 100,000 inhabitants, (b) concentration of BCCs between 8:00 am and I2:00 pm or between 2:00 pm and $6 \mathrm{pm}$, (c) greater frequency of alcoholic beverage consumption on weekends, (d) in relation to age, the 18-24 age group had the highest number of corrective measures.

Keywords: COVID-19, epidemic, confinement, public health, police

\section{RESUMO}

O objetivo deste trabalho foi identificar os padrões de espaço e temporários de sete comportamentos contrários à convivência (CCC) durante as medidas de confinamento aplicadas a nível nacional na Colômbia durante 47 dias. Para isto foram analisados 363439 registros administrativos de CCC coletados pela Polícia Nacional desde 16 de março a $1^{\circ}$ de maio de 2020, com a seguinte informação: hora, dia, data, tipo de comportamento, idade e departamento do infrator. Os resultados mostraram: (a) relações temporárias entre o número de casos diários ou acumulados de COVID-19 e as taxas de CCC por 100000 habitantes, (b) concentração das CCC entre as 08: 00 e as 12:00 horas ou entre as 14:00 e as 18:00 horas, (c) maior frequência de consumo de bebidas alcoólicas durante os fins de semana, (d) com relação à idade, a faixa de 18 a 24 anos é a que apresenta um maior registro de medidas corretivas.

Palavras-chave: COVID-19, epidemia, confinamento, saúde pública, policial

Ante el alto de riesgo de contagio por el COVID-19, los Estados han implementado diferentes acciones encaminadas a reducirlo. La cuarentena, el confinamiento social o el aislamiento social (véase Parmet \& Sinha, 2020, para una revisión de conceptos diferenciales) constituyen medidas frecuentes ante enfermedades epidémicas desde la Edad Media (De Foronda, I80 I; Von Justi, 1996), la gripe de 1918 (Tramullas, 2020), y hasta iniciado el siglo XXI con relación al ébola en varios países africanos y en Estados Unidos (Parmet \& Sinha, 2020), India (Paital, Das \& Kumar, 2020; Lamba, 2020), Francia (Salje et al., 2020), España o Colombia, con respecto al COVID-19. Así, los gobiernos nacionales y las autoridades locales han recurrido a expedir normas legales con el fin de regular dichas medidas, $y$ otras tales como las relativas al distanciamiento social, el uso de guantes, el uso de mascarillas o el tapabocas. En el caso de Colombia, estas disposiciones legales se enmarcan en el Código Nacional de Seguridad y Convivencia Ciudadana (Ley I80I de 29 de julio de 2016 y la Ley 2000 del I4 de noviembre de 2019).
En este marco, algunos autores cuestionan la efectividad del confinamiento social sobre la propagación del COVID-19 (Parmet \& Sinha, 2020), mientras que otros señalan su impacto positivo: en la India se considera esencial para la prevención frente a la carencia de una infraestructura sanitaria adecuada (Paital et al., 2020), y en Francia se estima que el confinamiento social ha logrado reducir el $77 \%$ de los contagios esperados (Salje et al., 2020). Ahora, estas medidas pueden disminuir su efectividad si la gente no se adhiere a su cumplimiento.

Entre las diferentes estrategias que permiten medir el incumplimiento de las leyes se encuentran los registros oficiales de criminalidad (Beirne \& Messerschmidt, 2006; Maguire, Morgan \& Reiner 2002; Ruiz, 20I4a). Una desventaja de este tipo de registros es que dependen de la voluntad de denunciar por parte de las víctimas o de los ciudadanos testigos de los hechos, así como de la eficacia de los controles policiales (e.g., controles vehiculares, requisas en la calle, etc.), de modo que pueden ocurrir delitos o infracciones que no llegan a conocimiento de las autoridades. De igual forma, no todas las conductas 
socialmente reprochables se refieren a delitos o crímenes, sino que existe otro tipo de conductas como, por ejemplo, las contravenciones administrativas o los comportamientos contrarios a la convivencia (CCC), de las cuales también se tienen registros administrativos, principalmente por la acción de la policía. En general, el volumen de investigación publicada y de teorización sobre los CCC no es tan amplio ni variado como lo que ocurre con los crímenes; sin embargo, los medios desempeñan hoy, además, un papel importante en sacar a la luz hechos o infracciones relacionados con los CCC (EI Tiempo, 24 de abril de 2020) debido a sus implicaciones en el riesgo de aceleración de los contagios.

Por otra parte, los registros oficiales, en este caso relacionados con las violaciones a los lineamientos nacionales sobre el comportamiento a seguir por la ciudadanía para evitar o reducir el contagio por COVID-19, permiten una sistematización uniforme del registro a nivel nacional, con cubrimiento de todas las regiones del país. En segundo lugar, permiten reunir un acumulado de registros que pueden analizarse desde una perspectiva censal $y$, en tercero, las posibles diferencias en la actuación por región de las diferentes unidades de la Policía encargadas de detectar los incumplimientos pueden constituirse en una variable en sí misma que se incluya en el análisis y la interpretación de los datos.

Este trabajo se enmarca en la necesidad de identificar factores de riesgo del incumplimiento de medidas de aislamiento y de auto y heterocuidado sanitario con relación a la crisis del COVID-19. Un primer paso en este sentido $-y$ lo que constituye el objetivo general de este trabajoes analizar los patrones espaciales, temporales y demográficos que presentan siete infracciones al Código Nacional de Seguridad y Convivencia Ciudadana que se pueden relacionar con el incumplimiento de las medidas adoptadas por los gobiernos nacional y locales orientadas a reducir el riesgo de contagio por el COVID-19. Se han tenido en cuenta para este trabajo siete tipos de comportamientos contrarios a la convivencia: (1) por aglomeración, (2) por incumplimiento de aislamiento social al violar el toque de queda, (3) por incumplimiento de la Ley Seca, (4) por violar restricciones de movilidad, al llevar un pasajero en vehículos motocicletas, (5) por incumplimiento de la restricción de salir a la calle según el género, (6) por violar la restricción de salir a la calle según el último número del documento de identidad, y (7) por no uso de tapabocas.

Estos comportamientos contrarios a la convivencia (CCC), los cuales en los anteriores códigos de Policía se conocían como contravenciones, corresponden al derecho administrativo de policía —no al penal- (Torres-Rico, 2008). Identificar estos patrones espacio-temporales y demográficos puede ayudar a orientar la actuación de las agencias de salud y policiales en el propósito de lograr una mayor efectividad de las normas y el nivel de conducencia que debe caracterizar a la ley para que esta pueda cumplirse (Muñoz-Sabaté, 1980).

\section{Metodología}

El presente estudio es de tipo exploratorio, tanto descriptivo como correlacional, ya que se pretende mostrar los valores que toman las variables que conforman la base de datos y analizar las relaciones entre ellas.

En cuanto a los participantes del estudio, se trata de un estudio censal, ya que se consideran todos los registros del Sistema del Registro Nacional de Medidas Correctivas (RNMC), de la Dirección de Seguridad Ciudadana (DISEC) de la Policía Nacional (2020a), en relación con las medidas de confinamiento de ámbito nacional. En el análisis de los datos es necesario tener en cuenta que, aunque la normativa sea de ámbito nacional, los gobernadores departamentales y las alcaldías municipales han tenido autonomía para expedir actos administrativos a fin de instaurar de forma obligatoria ciertas medidas, como, por ejemplo, el toque de queda, o el número de veces a la semana que las personas adultas están autorizadas para salir a la calle o la modalidad de dicha salida (e. g., por número de documento de identidad o por género). El tamaño de la población es de 363439 registros de comportamientos contrarios a la convivencia (CCC) desde el 16 de marzo del 2020 al I de mayo del 2020.

\section{Materiales e instrumentos}

El corpus de datos está conformado por la base a nivel nacional de CCC a las medidas decretadas que regulan el confinamiento. Esta base de datos la conforman, a su vez, las siguientes variables:

- Variables de localización. Departamento y municipio.

- Fechas del registro. Fecha de ocurrencia del CCC y número del día desde que comenzaron las sanciones; así, la base de datos registra medidas correctivas para 47 días.

- Número de la semana de ocurrencia. Los 47 días anteriores se agruparon en ciclos de siete días, con el fin de reducir los periodos de comparación de la ocurrencia de los CCC. Estas semanas no corresponden a los ciclos de 
lunes a domingo, sino a ciclos de siete días desde el 16 de marzo del 2020.

- Día de la semana de ocurrencia del hecho. De lunes a domingo.

- Hora del día del registro. Se presenta en horas en punto en el formato de 24 horas y en agrupamientos de cada dos horas, con el propósito de facilitar el análisis de los CCC según el momento del día en que ocurrieron.

- Tipo. Tiene siete modalidades: (I) aglomeración de personas, (2) aislamiento obligatorio por toque de queda, (3) consumo de bebidas embriagantes en ley seca, (4) restricción a la movilidad (parrillero), (5) restricción por género, (6) restricción por número de documento, y (7) uso de tapabocas.

- Datos sociodemográficos. Son la edad, la nacionalidad y la condición de nacionalidad (nacional colombiano, residente o extranjero). Los registros de los CCC no incluyen variables tales como el sexo o el estrato.

\section{Procedimiento y plan de análisis}

La información del Registro Nacional de Medidas Correctivas durante las medidas de aislamiento fue proporcionada por el Grupo del Registro Nacional de Medidas Correctivas de la Dirección de Seguridad Ciudadana (Policía Nacional, 2020a), con autorización para su revisión y procesamiento con fines de análisis de los datos. Estos consistieron en análisis de frecuencias de las variables, la elaboración de tablas de contingencia entre variables categóricas y la comparación de medias entre variables categóricas y la edad.

\section{Resultados}

\section{Datos generales}

La edad va de los 14 a los 80 años, encontrándose una media de 30,I años y una desviación típica de 10,68. Se procedió a una agrupación de la edad en siete grupos: desde los 14 a los 17 años (I, I 3\%), de los 18 a los 24 (36,99\%), de los 25 a los 30 (25,01\%), de los 31 a los 40 (2I,06\%), de los $4 \mathrm{I}$ a los 50 (9,54\%), de los $5 \mathrm{I}$ a los $60(4,64 \%)$ y de los $6 \mathrm{I}$ a los 80 años ( $1,53 \%)$.

La tabla I presenta los resultados relacionados con la nacionalidad. La mayoría de los CCC son atribuibles a nacionales colombianos/as, seguidos en segundo lugar por personas de Venezuela y, con porcentajes del $0,01 \%$ o superiores, aparecen personas de Ecuador (66 casos), de Estados Unidos (54), Perú (35), Brasil (29) yArgentina (20). Con porcentajes menores se encuentran sujetos de otras 34 nacionalidades, con un único participante (e.g., Australia) a algo más de una decena: Francia (I0 casos), Alemania (II) o España (14). Por otra parte, si se agrupan los registros según el tipo de documento de identidad, se encuentra que la mayoría son nacionales colombianos/as (personas con cédula de ciudadanía o tarjeta de identidad para menores de edad), seguidos de los extranjeros residentes en Colombia, con cédula de extranjería y los extranjeros sin residencia.

Tabla I. Datos generales de la muestra

\begin{tabular}{|c|c|c|c|}
\hline Nacionalidad & $\%$ & Nacionalidad & $\%$ \\
\hline Colombia & 88,29 & Perú & 0,01 \\
\hline Venezuela & $|I, 6|$ & Brasil & 0,01 \\
\hline Ecuador & 0,02 & Argentina & 0,01 \\
\hline Estados Unidos & 0,01 & Otros & 0,04 \\
\hline \multicolumn{4}{|c|}{ Residencia } \\
\hline $\begin{array}{l}\text { Ciudadanía } \\
\text { colombiana }\end{array}$ & $\begin{array}{c}\text { Extranjero } \\
\text { residente }\end{array}$ & $\begin{array}{r}\text { Extra } \\
\text { no res }\end{array}$ & $7,06 \%$ \\
\hline
\end{tabular}

\section{Dinámicas territoriales de los CCC}

A nivel departamental se puede considerar bien sea las frecuencias absolutas o bien las ponderadas en razón a la cantidad de población por las regiones de Colombia. Así, la tabla 2 muestra el total de casos por departamento en términos absolutos y por cada tipo de CCC, y la tabla 3 presenta las tasas de casos departamentales por cada 100000 habitantes. Esta distinción entre casos absolutos y tasas por 100000 habitantes es relevante, porque para el total de casos absolutos aparecen con mayor número de infracciones los departamentos con mayor población, además de Bogotá, Antioquia, Atlántico, Valle del Cauca, Santander y Nariño. En cambio, el indicador de número de casos por cada 100000 habitantes muestra que las tasas del total de CCC se dan en departamentos con menor población y dificultades de acceso, Amazonas, Guainía y Vichada, junto con otros como, por ejemplo, Casanare y Putumayo.

En cuanto al tipo de comportamiento y su tasa de ocurrencia entre el total de registros, el análisis de los datos muestra que la mayoría de las infracciones corresponde a violación del aislamiento obligatorio por toque de queda, con el $\mathbf{8 7 , 4 3 \%}$ de los casos, seguido de la restricción por número de documento $(5,36 \%)$, la restricción por razón de género (2,56\%), el consumo de bebidas embriagantes ( $1,86 \%)$, la aglomeración de personas $(1,47 \%)$, la restricción de movilidad por llevar parrillero/a $(0,94 \%)$ y por no usar tapabocas $(0,39 \%)$. 
Tabla 2. Número de casos absolutos de CCC por departamento y Bogotá y tipo de infracción

\begin{tabular}{|c|c|c|c|c|c|c|c|c|}
\hline Departamento & Total & AGLO & TOQUE & CONAL & MOVIL & GENE & DOCU & TABO \\
\hline Amazonas & 925 & 0 & 701 & 1 & 85 & 0 & 138 & 0 \\
\hline Antioquia & 53428 & 439 & 50354 & 330 & 592 & 8 & 1518 & 187 \\
\hline Arauca & 1215 & 3 & 1026 & 85 & 2 & 0 & 93 & 6 \\
\hline Atlántico & 24615 & 562 & 21842 & 241 & 137 & 5 & 1622 & 206 \\
\hline Bolívar & 8925 & $|5|$ & 7175 & 78 & 110 & 743 & 646 & 22 \\
\hline Boyacá & 9498 & 178 & 8078 & 774 & 42 & 6 & 411 & 9 \\
\hline Caldas & 5551 & 98 & 5263 & 68 & 47 & 0 & 75 & 0 \\
\hline Caquetá & 3992 & 53 & 2644 & 35 & 2 & 1 & 247 & 20 \\
\hline Casanare & 4084 & 27 & 3317 & 47 & 28 & 11 & 646 & 8 \\
\hline Cauca & 9037 & 65 & 8311 & 60 & 158 & 0 & 422 & 21 \\
\hline Cesar & 9204 & 74 & 7973 & 148 & 90 & 202 & 599 & 118 \\
\hline Chocó & 2012 & 9 & 1934 & 18 & 31 & 2 & 18 & 0 \\
\hline Córdoba & 6386 & 25 & 6012 & 68 & 6 & 1 & 270 & 4 \\
\hline Cundinamarca & 1187 & 337 & $|6| 4 \mid$ & 648 & 86 & 186 & 1738 & 51 \\
\hline Guainía & 1180 & 0 & 1175 & 4 & 1 & 0 & 0 & 0 \\
\hline Guaviare & 600 & 0 & 575 & 20 & 4 & 0 & I & 0 \\
\hline Huila & 9253 & 47 & 6522 & 68 & 244 & 29 & 2330 & 13 \\
\hline La Guajira & 6282 & 37 & 6044 & 28 & 36 & 5 & 131 & 1 \\
\hline Magdalena & 9732 & 127 & 8765 & 161 & 244 & 1 & 379 & 55 \\
\hline Meta & 7598 & 75 & 6013 & 235 & 180 & 257 & 829 & 9 \\
\hline Nariño & 10291 & 156 & 8770 & 425 & 166 & 8 & 729 & 37 \\
\hline Norte Santander & 9895 & 147 & 7936 & 175 & 5 & 14 & 1493 & 65 \\
\hline Putumayo & 3872 & 54 & 3194 & 36 & 202 & 3 & 380 & 3 \\
\hline Quindío & 4559 & 23 & 4501 & 7 & 3 & 4 & 21 & 0 \\
\hline Risaralda & 9499 & 9 & 9044 & 15 & 8 & 0 & 421 & 2 \\
\hline San Andrés & 471 & 3 & 438 & 23 & 2 & 0 & 4 & 1 \\
\hline Santander & $|775|$ & 174 & 15435 & 302 & 76 & 1 & 1735 & 28 \\
\hline Sucre & 5994 & 7 & 5576 & 71 & 112 & 0 & 168 & 10 \\
\hline Tolima & 8493 & 93 & 7457 & 139 & 122 & 15 & 643 & 24 \\
\hline Valle del Cauca & 23011 & 409 & 20366 & 260 & 163 & 25 & 1728 & 60 \\
\hline Vaupés & 345 & 0 & 345 & 0 & 0 & 0 & 0 & 0 \\
\hline Vichada & 773 & 2 & 730 & 13 & 11 & 0 & 17 & 0 \\
\hline Bogotá & 7677| & 1904 & 64087 & 2163 & 361 & 7776 & 28 & 452 \\
\hline
\end{tabular}

Nota: AGLO: sanciones por aglomeración; TOQUE: sanciones por violación del toque de queda; CONAL: sanciones por consumo de alcohol en ley seca; MOVIL: violación de la restricción de movilizarse en moto sin acompañante; GENE: violar la restricción de salir a la calle por géneros; DOCU: sanciones por salir a la calle en día diferente al autorizado según número final del documento de identificación;TAPA: sanciones por no uso del tapabocas.

En las tablas 2 y 3 se muestran las frecuencias de CCC y el número de casos por cada 100000 habitantes $\times 1000$, por departamentos, tomando las cifras departamentales de la población para el 2018 (Departamento Administrativo Nacional de Estadística [DANE], 23 de mayo de 2020).
Para el total de casos ponderados por 100000 habitantes se destacan con mayor participación Bogotá y los departamentos de Antioquia,Atlántico,Valle del Cauca y Nariño, mientras que los casos con menos casos se encuentran en el Amazonas, Guaviare, San Andrés, Vaupés y Vichada. 
Tabla 3. Tasas de CCC por 100000 habitantes por departamento y Bogotá y tipo de infracción

\begin{tabular}{|c|c|c|c|c|c|c|c|c|}
\hline Departamento & TOTAL & AGLO & TOQUE & CONAL & MOVIL & GENE & DOCU & TAPA \\
\hline Amazonas & || $73,4 \mid$ & 0,00 & 889,26 & $\mathrm{I}, 27$ & 107,83 & 0,00 & 175,06 & 0,00 \\
\hline Antioquia & 798,51 & 6,56 & 752,57 & 4,93 & 8,85 & 0,12 & 22,69 & 2,79 \\
\hline Arauca & 448,82 & $\mathrm{I}, \mathrm{II}$ & 379,01 & 31,40 & 0,74 & 0,00 & 34,35 & 2,22 \\
\hline Atlántico & 966,76 & 22,07 & 857,85 & 9,47 & 5,38 & 0,20 & 63,70 & 8,09 \\
\hline Bolívar & $4 I I, 00$ & 6,95 & 330,41 & 3,59 & 5,07 & 34,22 & 29,75 & $\mathrm{I}, 0 \mathrm{I}$ \\
\hline Boyacá & 740,89 & 13,88 & 630,12 & 60,38 & 3,28 & 0,47 & 32,06 & 0,70 \\
\hline Caldas & 558,52 & 9,86 & 529,55 & 6,84 & 4,73 & 0,00 & 7,55 & 0,00 \\
\hline Caquetá & 804,41 & 10,68 & 532,78 & 7,05 & 0,40 & 0,20 & 49,77 & 4,03 \\
\hline Casanare & 1088,32 & 7,20 & 883,93 & 12,52 & 7,46 & 2,93 & 172,15 & 2,13 \\
\hline Cauca & 638,14 & 4,59 & 586,87 & 4,24 & 11,16 & 0,00 & 29,80 & $\mathrm{I}, 48$ \\
\hline Cesar & 863,71 & 6,94 & 748,19 & 13,89 & 8,45 & 18,96 & 56,21 & II,07 \\
\hline Chocó & 390,55 & $\mathrm{I}, 75$ & 375,41 & 3,49 & 6,02 & 0,39 & 3,49 & 0,00 \\
\hline Córdoba & 357,03 & $\mathrm{I}, 40$ & 336,12 & 3,80 & 0,34 & 0,06 & 15,10 & 0,22 \\
\hline Cundinamarca & 42,33 & 12,02 & 575,59 & 23,11 & 3,07 & 6,63 & 61,98 & 1,82 \\
\hline Guainía & 2716,02 & 0,00 & 2704,51 & 9,21 & 2,30 & 0,00 & 0,00 & 0,00 \\
\hline Guaviare & 518,00 & 0,00 & 496,42 & 17,27 & 3,45 & 0,00 & 0,86 & 0,00 \\
\hline Huila & 772,98 & 3,93 & 544,84 & 5,68 & 20,38 & 2,42 & 194,65 & 1,09 \\
\hline La Guajira & 749,44 & 3,56 & 581,05 & 2,69 & 3,46 & 0,48 & 12,59 & 0,10 \\
\hline Magdalena & 747,34 & 9,78 & 674,98 & 12,40 & 18,79 & 0,08 & 29,19 & 4,24 \\
\hline Meta & 568,78 & 7,38 & 591,44 & 23,11 & 17,70 & 25,28 & 81,54 & 0,89 \\
\hline Nariño & 711,17 & 8,62 & 484,72 & 23,49 & 9,17 & 0,44 & 40,29 & 2,04 \\
\hline Norte Santander & 1078,86 & 10,57 & 570,37 & 12,58 & 0,36 & $\mathrm{I}, 0 \mathrm{I}$ & 107,30 & 4,67 \\
\hline Putumayo & 792,92 & 15,05 & 889,95 & 10,03 & 56,28 & 0,84 & 105,88 & 0,84 \\
\hline Quindío & 981,52 & 4,00 & 782,84 & 1,22 & 0,52 & 0,70 & 3,65 & 0,00 \\
\hline Risaralda & 600,67 & 0,93 & 934,51 & $\mathrm{I}, 55$ & 0,83 & 0,00 & 43,50 & 0,21 \\
\hline San Andrés & 848,98 & 3,83 & 558,58 & 29,33 & 2,55 & 0,00 & 5,10 & 1,28 \\
\hline Santander & 683,45 & 8,32 & 738,22 & 14,44 & 3,63 & 0,05 & 82,98 & 1,34 \\
\hline Sucre & 598,12 & 0,80 & 635,79 & 8,10 & 12,77 & 0,00 & 19,16 & 1,14 \\
\hline Tolima & 483,86 & 6,55 & 525,16 & 9,79 & 8,59 & 1,06 & 45,28 & 1,69 \\
\hline Valle del Cauca & 767,90 & 8,60 & 428,24 & 5,47 & 3,43 & 0,53 & 36,33 & 1,26 \\
\hline Vaupés & 1000,31 & 0,00 & 767,90 & 0,00 & 0,00 & 0,00 & 0,00 & 0,00 \\
\hline Vichada & 938,40 & 2,59 & 944,67 & 16,82 & 14,23 & 0,00 & 22,00 & 0,00 \\
\hline Bogotá & 695,16 & 23,27 & 783,36 & 26,44 & 4,41 & 95,05 & 0,34 & 5,52 \\
\hline
\end{tabular}

Nota: AGLO: sanciones por aglomeración; TOQUE: sanciones por violación del toque de queda; CONAL: sanciones por consumo de alcohol en ley seca; MOVIL: violación de la restricción de movilizarse en moto sin acompañante; GENE: violar la restricción de salir a la calle por géneros; DOCU: sanciones por salir a la calle en día diferente al autorizado según número final del documento de identificación;TAPA: sanciones por no uso del tapabocas.

Con esto se puede apreciar que el número absoluto de casos hace sobresalir a los departamentos más poblados y a Bogotá, pero la tasa de infracciones por cada 100000 $X 1000$ habitantes refleja un panorama diferente que se complejiza aún más cuando se analizan la distribución de casos por cada tipo de CCC. Así, por aglomeración son más frecuentes en Bogotá,Atlántico y Putumayo, con menos casos se encuentran Guainía, Guaviare y Vaupés, mientras que Arauca, Sucre y Risaralda presentan cero casos. 
En cuanto al toque de queda se presentaron más casos en Guainía, Vichada, Risaralda, Amazonas y Casanare, así como menores tasas en Arauca, Bolívar, Chocó y Córdoba. Con relación al consumo de bebidas alcohólicas, se destaca con las mayores tasas el eje cundiboyacense junto con Arauca, Meta, Nariño, San Andrés y Bogotá, mientras que los coeficientes más bajos se dan en Amazonas, La Guajira, Quindío, Risaralda y Vaupés.

Siguiendo a la movilidad por llevar parrillero, las tasas son más altas en el Amazonas, Putumayo, Cauca y Huila, mientras que son más bajas en Arauca, Caquetá, Córdoba, Norte de Santander, Quindío, Risaralda y Vaupés. Por su parte, los CCC por las restricciones de género se dieron especialmente en Bogotá, Bolívar, Meta y el Cesar, con diez departamentos sin ningún caso, mientras que las tasas por restricción de documentos se dieron, sobre todo, en el Amazonas, Casanare, Huila, Norte de Santander y Putumayo, con menores tasas en Bogotá, Caldas, Chocó, Guaviare, Quindío y San Andrés. Por último, con relación al uso de tapabocas, la tasa mayor fue en los departamentos de Atlántico, Cesar, Norte de Santander y Bogotá, frente a tasas más bajas en Bolívar, Cauca, La Guajira, Meta, Putumayo y Risaralda.

\section{Dinámicas temporales}

Si se analiza la distribución por cada día, se obtienen patrones de distribución temporal de cada CCC, los cuales se pueden cruzar con la evolución de los propios casos de COVID-19. Por una parte, en las figuras I y 2 se muestra la distribución por toque de queda — que constituye la mayoría, en la figura I-y por las otras seis, en la figura 2. Respecto al toque de queda se aprecian unos picos superiores al inicio de la tercera semana y una tendencia al decremento paralela a la acumulación de casos detectados de personas infectadas por el COVID-19'.

Los demás tipos de incumplimientos no se relacionaron con el número de casos acumulados de COVID-19, pero sí con el conteo diario de los contagios detectados

Así, para los CCC por aglomeración se aprecia un decremento de las infracciones y a la par un aumento de los registros hacia los días 29 ○ 30, y el mismo patrón se advierte para los CCC por movilidad con parrillero. Por su parte, con relación a las sanciones por el no uso de tapabocas y violar las restricciones de salir por el número de cédula, se aprecia una cierta relación positiva: con el paso de los días crece el número de sanciones por dichos comportamientos y también lo hace el número de casos diarios de infectados por COVID-19. En cambio, para los CCC por restricción de género o de consumo de alcohol no se identifica un patrón de casos progresivo o regresivo por número de día, si bien los saltos que se aprecian por consumo de alcohol sugieren una cierta periodicidad quizá asociada al día de la semana. A fin de comprobar esto, se cruzó el tipo de infracción con el día de la semana (de lunes a domingo).

De acuerdo con el día de la semana (véase la tabla 4), se puede apreciar que las medidas correctivas por aglomeración se incrementan de manera progresiva desde el martes al domingo, las infracciones al toque de queda parecen ser

I Los datos de infectados diarios se encuentran, desde el 18 de marzo, en: https://www. google.com/search?client=firefox-b-d\&q=n\%C3\%BAmero+de+infectados+por+coro navirus+en+colombia. Recuperado el 15 de mayo del 2020.

Evolución de CCC al toque de queda y acumulación de casos por COVID-19

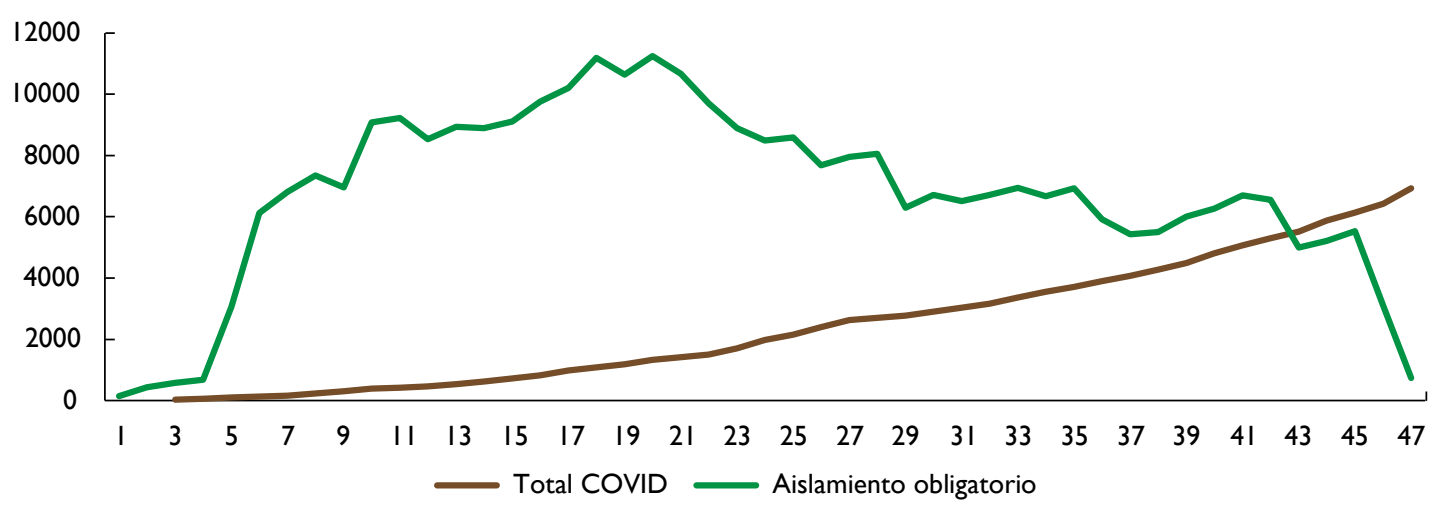

Figura I. Distribución del número de CCC por toque de queda a lo largo de 47 días. 

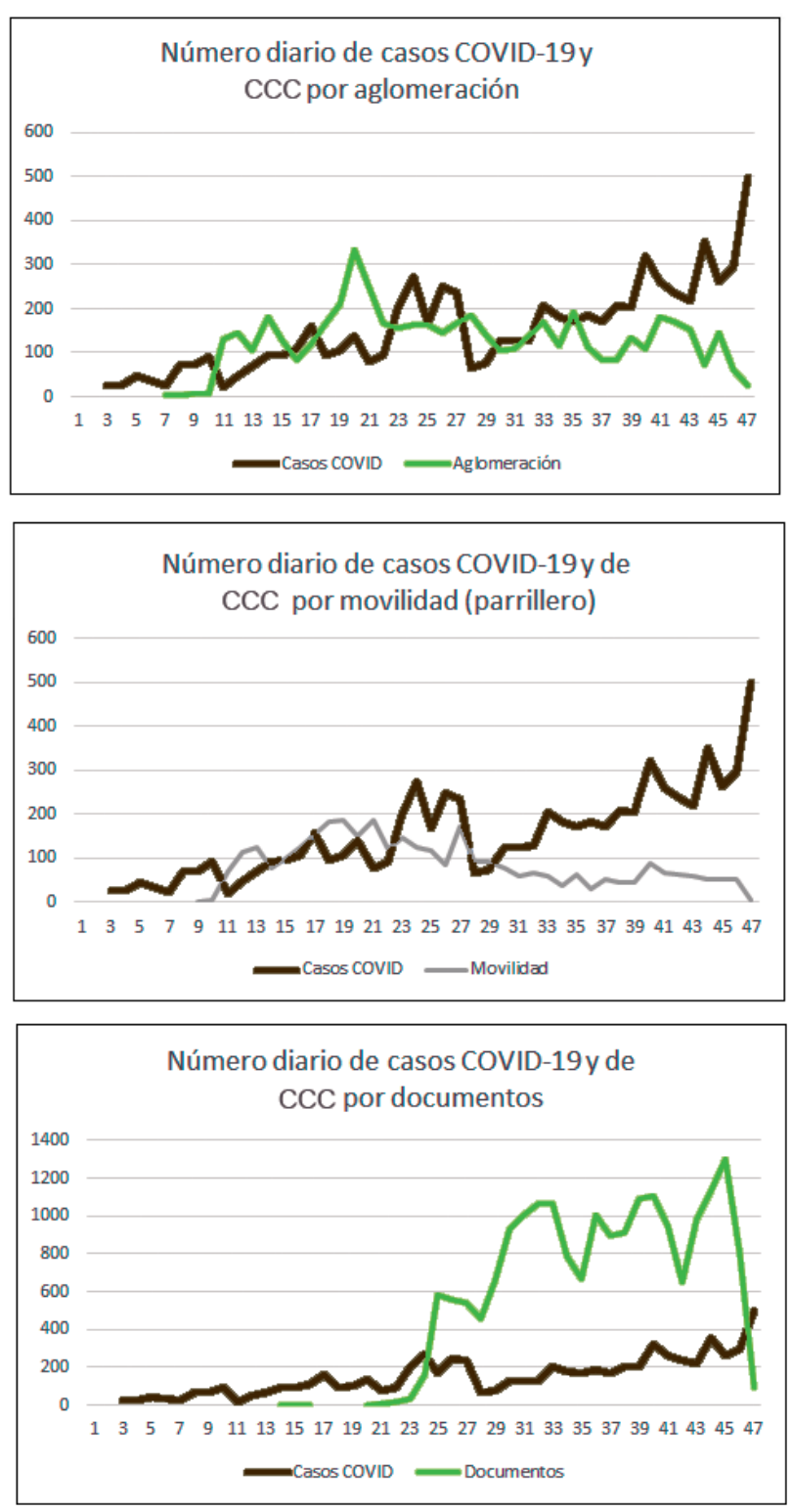

Figura 2. Distribución de CCC y número de casos diarios de COVID-19.

algo mayores el domingo y algo más bajas el lunes y el martes, las de consumo de alcohol se concentran los sábados y los domingos ( $y$ menos los lunes y martes), las de movilidad se incrementan los jueves, los viernes y los sábados, con menos casos el lunes; las medidas correctivas por las restricciones de género son menores el fin de semana (sábado y domingo), lo mismo que por violación de la restricción de documentos. Por otra parte, por no uso de tapabocas se han dado más los días martes y miércoles.

A continuación, se exploró la distribución de los CCC a lo largo de las 24 horas del día (véase la figura 3 ) encontrándose diferencias entre periodos del día, con
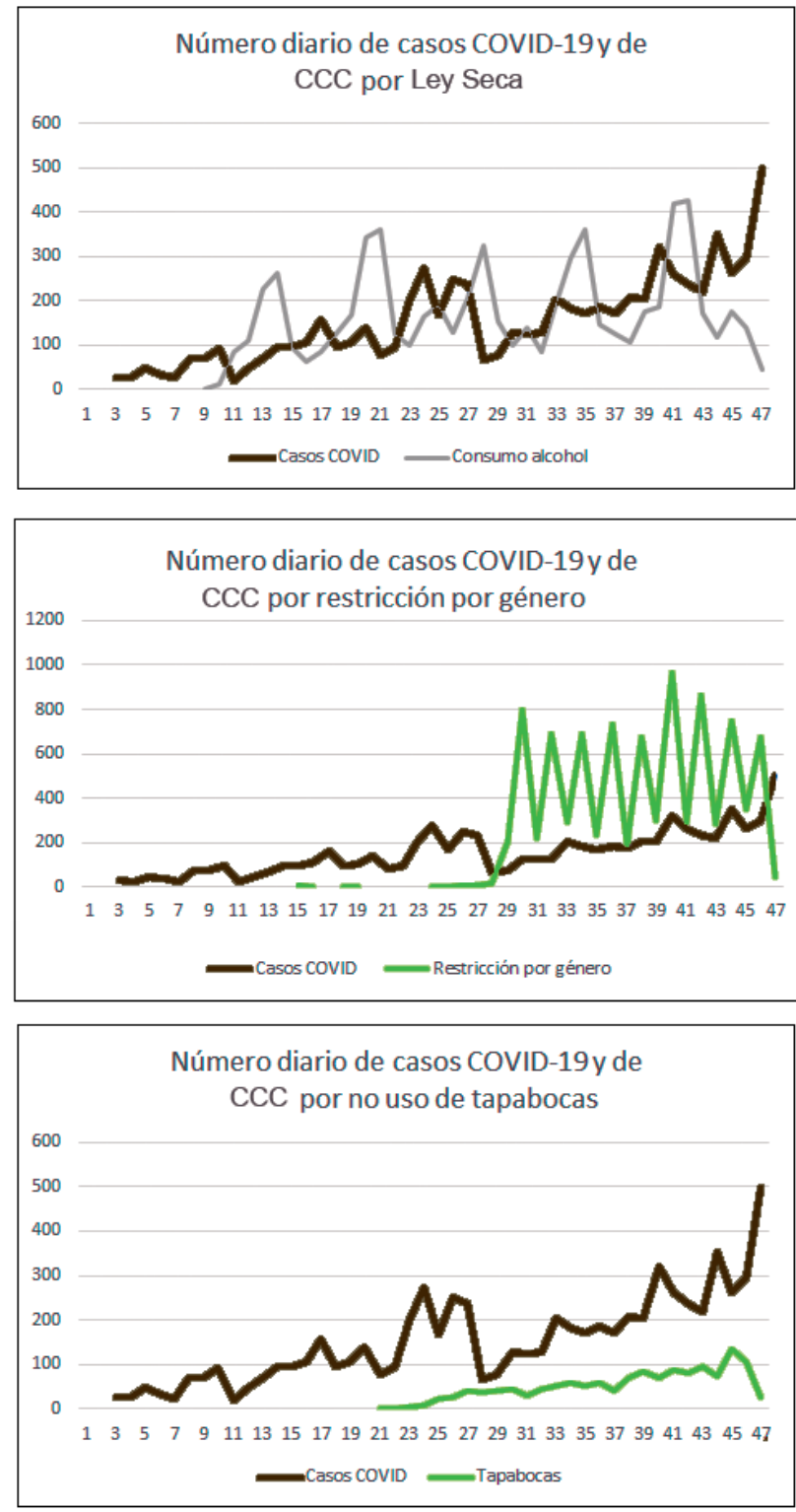

un $\chi^{2}(66): 6355, I p<0,001$. Se puede apreciar que las sanciones por aglomeración de personas se concentran entre las 8: 00 am y las 12 del mediodía, y entre las 2:00 y las 8: p. m. Un patrón similar siguen las demás con algunas particularidades: por ejemplo, las medidas correctivas por consumo de bebidas embriagantes se concentran desde las 4: 00 p. m. en adelante, incluyendo horas de la madrugada, desde las 00 horas a las 4:00 a. m.

En cuanto a la edad, los CCC, en general, se concentran en las edades de los 18 a los 40 años, y especialmente en la franja de 18 a 24 años, en seis de los comportamientos 
Tabla 4. Distribución de comportamientos contrarios a la convivencia por día de la semana

\begin{tabular}{|c|c|c|c|c|c|c|c|}
\hline & Aglomeración & Toque de queda & Ley Seca & $\begin{array}{c}\text { Movilidad } \\
\text { (parrillero) }\end{array}$ & $\begin{array}{l}\text { Restric. } \\
\text { Género }\end{array}$ & $\begin{array}{c}\text { Restric. } \\
\text { Documen. }\end{array}$ & Uso de tapabocas \\
\hline Lunes & 13,19 & 13,70 & 10,23 & 11,83 & 13,17 & 13,73 & 14,02 \\
\hline Martes & 9,54 & 13,66 & 7,47 & 13,32 & 18,76 & 15,34 & 11,76 \\
\hline Miércoles & 11,80 & 14,44 & 10,17 & 12,97 & 13,47 & 17,39 & $|7,7|$ \\
\hline Jueves & 14,89 & 14,32 & 11,95 & 15,60 & 17,96 & 18,18 & 18,27 \\
\hline Viernes & 15,14 & $|3,8|$ & 12,32 & 15,90 & 14,08 & 14,50 & 12,39 \\
\hline Sábado & 16,99 & 14,99 & 22,09 & 16,16 & 10,59 & 11,68 & 13,67 \\
\hline Domingo & 18,45 & 15,09 & 25,78 & 14,23 & 11,97 & 9,18 & 12,18 \\
\hline Chi2 & $163,9 * * *$ & $247,6 * * *$ & $1168,2 * * *$ & $31,2 * * *$ & $433,8 * * *$ & $945,1 * * *$ & $38,1 * * *$ \\
\hline
\end{tabular}

Distribución de las contravenciones por ciclos horarios

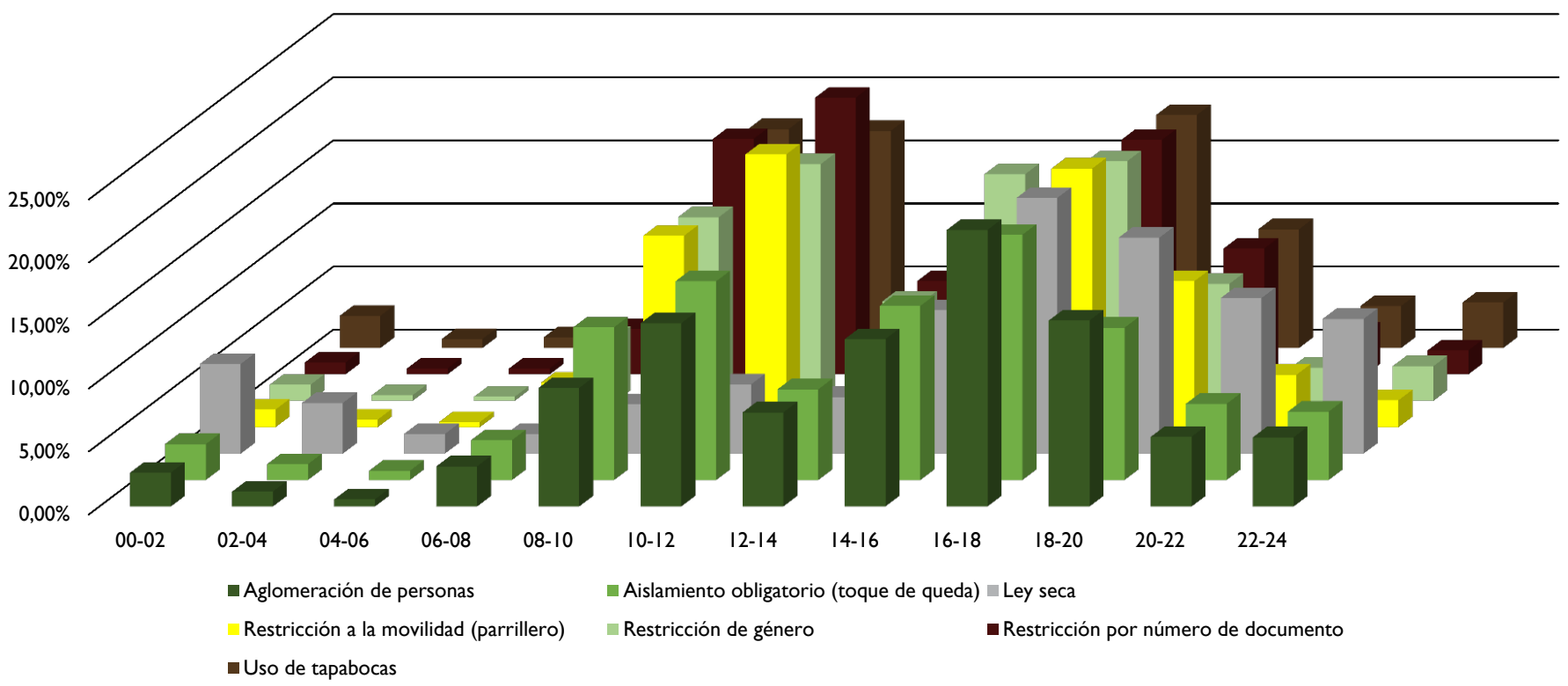

Figura 3. Distribución de horarios de los comportamientos contrarios a la convivencia a las medidas contra el COVID-I9

contrarios a la convivencia; la del consumo de bebidas alcohólicas en Ley Seca es la que muestra una dispersión más similar entre los segmentos de edad, incluyendo un $17,07 \%$ de sujetos sancionados por consumo de alcohol entre los 41 y los 50 años (véase tabla 5).

\section{Discusión y conclusiones}

En el presente trabajo, las unidades de análisis son tanto los registros individuales de siete tipos de comportamientos contrarios a la convivencia al Código Nacional de Seguridad y Convivencia Ciudadana como los momentos en que ocurren, a nivel de número de día desde el primer registro en la base de datos, el día de la semana y la hora de ocurrencia. A nivel espacial, la unidad de análisis que se tomó fue el departamento en el que registró la medida correctiva. Esto supone adoptar una perspectiva propia de la criminología comparada, en la medida en que esta estudia las diferencias entre contextos, como, por ejemplo, las regiones o los países en las tasas del crimen (Beirne \& Messerschmidt, 2006). Esta perspectiva comparada se relaciona con la del análisis espacial del crimen (Aksoy, 2017; Jendryke \& McClure, 2019; Ruiz, 20I4b). Por último, a nivel demográfico, la edad del infractor fue la variable incluida en la base de datos y en los análisis del presente estudio. 
Tabla 5. Distribución de los comportamientos contrarios a la convivencia por grupos de edad

\begin{tabular}{|c|c|c|c|c|c|c|c|}
\hline $\begin{array}{l}\text { Edad en } \\
\text { intervalos }\end{array}$ & $\begin{array}{l}\text { Aglomeración } \\
\text { de personas }\end{array}$ & $\begin{array}{c}\text { Toque } \\
\text { de queda }\end{array}$ & Ley Seca & $\begin{array}{l}\text { Restricción movilidad } \\
\text { (parrillero) }\end{array}$ & $\begin{array}{l}\text { Restricción } \\
\text { de género }\end{array}$ & $\begin{array}{l}\text { Restricción número } \\
\text { de documento }\end{array}$ & $\begin{array}{l}\text { Uso de } \\
\text { tapabocas }\end{array}$ \\
\hline De 14 a 17 años & $0,88 \%$ & $\mathrm{I}, 23 \%$ & $0,27 \%$ & $0,85 \%$ & $0,18 \%$ & $0,40 \%$ & $0,64 \%$ \\
\hline De 18 a 24 años & $35,92 \%$ & $37,49 \%$ & $20,66 \%$ & $34,01 \%$ & $38,29 \%$ & $34,66 \%$ & $38,03 \%$ \\
\hline De 25 a 30 años & $24,65 \%$ & $25,09 \%$ & $22,86 \%$ & $24,19 \%$ & $26,12 \%$ & $23,99 \%$ & $26,06 \%$ \\
\hline De 3 I a 40 años & $21,22 \%$ & $20,94 \%$ & $25,88 \%$ & $23,78 \%$ & $21,17 \%$ & $22,59 \%$ & $21,03 \%$ \\
\hline De 4 I a 50 años & $10,44 \%$ & $9,27 \%$ & $17,07 \%$ & $10,93 \%$ & $9,15 \%$ & $11,18 \%$ & $7,86 \%$ \\
\hline De 5 I a 60 años & $4,46 \%$ & $4,51 \%$ & $9,60 \%$ & $4,80 \%$ & $3,97 \%$ & $5,44 \%$ & $4,82 \%$ \\
\hline De 61 a 80 años & $2,42 \%$ & $1,46 \%$ & $3,66 \%$ & $\mathrm{I}, 44 \%$ & $1,12 \%$ & I,74\% & $\mathrm{I}, 56 \%$ \\
\hline$\chi^{2}$ & $38,1 * * *$ & $809,2 * * *$ & $1603 * * *$ & $30,1 * * *$ & $105,9 * * *$ & $247.4 * * *$ & 8,39 n.s \\
\hline
\end{tabular}

**** $p<0,001$

Como resultados principales se haya cierta convergencia de varios tipos de infracciones en ciertos contextos: Putumayo presenta tasas altas en tres tipos de CCC (toque de queda, movilidad en motocicleta con segundo ocupante y en restricción de movilidad por número de documento de identificación). Este departamento sufrió inundaciones y un terremoto en su capital en el 2018, lo cual agravó las condiciones de pobreza de la región, en la que convergen las protestas sociales por falta de ayudas, la presencia de cultivos de coca y de intentos estatales para su erradicación, los enfrentamientos de campesinos con fuerzas policiales, entre otros (Arenas, 2020). Amazonas y Atlántico presentan altas tasas en dos tipos de infracción: violación del toque de queda y de la restricción por documentos en el Amazonas, y aglomeración y uso de tapabocas en el Atlántico. A fecha del 28 de abril del 2020 el Amazonas era el departamento con mayor tasa de contagios de COVID- 19 por cada 100000 habitantes, y se han realizado pronunciamientos de organizaciones indígenas por la falta de recursos sanitarios para atender esta población (Revista Semana, 21 de mayo de 2020). Además, esta área es limítrofe con la amazonia brasileña, la cual en aquel país ha sido una de las zonas más afectadas por el COVID-19. Para el caso del Atlántico, las altas tasas de aglomeración y no uso de tapabocas podrían relacionarse con el carácter turístico de la ciudad, especialmente con relación a celebraciones culturales como los carnavales. La tasa de incumplimiento de las medidas de aislamiento en la capital del Atlántico, Barranquilla, y en el área metropolitana, habría llegado al 7\% (Herrera, 17 de abril de 2020). En otros municipios del departamento del Atlántico, como, por ejemplo, Soledad, se habría llegado a un incumplimiento del confinamiento del $50 \%$, lo que llevó a considerar la militarización de la ciudad a finales de abril del 2020 (Caracol Radio Barranquilla, 21 de mayo de 2020).

También este carácter de región turística y su cultura asociada a celebraciones y fiestas podría ser uno de los factores explicativos de tasas altas de CCC en departamentos como el Cesar (tasas altas por restricción por género y en el uso de tapabocas), San Andrés y Boyacá (por consumo de bebidas alcohólicas en ley seca) o Bolívar, cuya capital, Cartagena, es uno de los principales destinos de turismo nacional e internacional del país, por violación a las restricciones por género. El caso de Boyacá es paradigmático en cuanto al consumo de alcohol, debido a que es el departamento que figura a la cabeza de las regiones colombianas en consumo de cerveza (Caracol Tunja, 2018, Revista Semana, s/f.), con varios municipios que presentan las mayores tasas de consumo de todo el país (Ávila-Jiménez, 2019; Pérez-Gómez, Lanziano, ReyesRodríguez, Mejía-Trujillo \& Cardozo-Macías, 2018).

Por otra parte, los resultados recolectados muestran patrones variados según infracción y unidad de tiempo considerada. En este sentido, la literatura muestra que se pueden hallar ciclos de tiempo tales como años, semanas o días en la manifestación de la criminalidad (Andresen \& Malleson, 20I5; Ashby \& Bowers, 2013). En el presente estudio, a lo largo de los días se advierte un decremento de las medidas correctivas por CCC, en conjunto y en tres tipos específicos: aglomeración, toque de queda y movilización con acompañante en motocicleta. Este decremento, que se da hacia la mitad del periodo cubier- 
to por los datos, parece paralelo a un incremento de los casos acumulados o diarios detectados de COVID-19 (véase la figura 2). Sin embargo, esta relación puede ser solo coincidente y podría basarse en el incremento de la capacidad de las autoridades colombianas de detectar nuevos casos de un lado o en que el 6 de abril de los corrientes la Dirección Nacional de la Policía Nacional emitiera la Circular 007 (2020b) en la que se establece y regula el aislamiento preventivo en reserva estratégica, como acto de servicio para el personal policial, lo que supuso desde el 8 de abril que un $20 \%$ del personal policial - según el tipo de unidad - fuera confinado en sus viviendas por turnos de 14 días. Esto ha podido conducir a un decremento en el número de efectivos policiales en las calles para vigilar el respeto a las medidas asociadas al distanciamiento social, así como podría haber repercutido en una disminución en el número de sanciones. También se puede plantear que la acción de vigilancia de la Policía con menos efectivos en las calles haya priorizado la vigilancia y sanción de ciertos CCC que van tomando importancia en el contexto del riesgo de contagio por COVID-19.Así, se aprecia un incremento paulatino de medidas correctivas relacionadas con las restricciones de salidas según el número de documento y por no uso de tapabocas (véase la figura 2). De igual forma, se puede plantear un quehacer policial dirigido súbitamente a este tipo de medidas de CCC en atención a los actos administrativos de los niveles nacional y local,y posteriormente a medida que se flexibilizan las medidas a volcar de nuevo su accionar en la persecución del delito y la atención tradicional de motivos policiales como, por ejemplo, la violencia doméstica. Finalmente, es posible señalar situaciones tales como la resistencia de ciertos sectores de la población a acatar las medidas, la violencia contra los servidores públicos y abstenerse de impartir medidas ante la presión ciudadana o de los medios de comunicación. A 19 de mayo del 2020 (ElTiempo, 2020a), la mayoría de las personas sancionadas no habría pagado la multa correspondiente por diferentes razones (e.g., no disponer del dinero, no saber dónde pagar por estar cerradas muchas oficinas, etc.) lo cual podría desalentar a ejercer ciertos aspectos de su función de control social, a lo que se une los cambios permanentes a nivel nacional y local en la regulación de actividades permitidas o no durante el confinamiento.

Sin embargo, es necesario señalar que para el mismo periodo de tiempo comparativo 2019-2020, 16 de marzo al I de mayo del 2020, en los registros de delitos del Sistema Estadístico Delictivo y Operativo de la Policía Nacional (2020c), todos los delitos de impacto social se reduje- ron en un $70,56 \%$, destacando reducciones como las del homicidio en un $37,53 \%$ ( 608 casos menos), los hurtos comunes en un $71,77 \%$ (37 I44 casos menos) o los hurtos de vehículos en un 74,28\% (4193 casos menos). Esto es indicador de que las medidas de aislamiento tuvieron un efecto dramático en la criminalidad registrada en el país, lo cual contribuye, sin lugar a dudas, a una temática de estudio internacional relacionada con la disminución en los datos de criminalidad en periodos de aislamiento.

En cuanto a los ciclos semanales, se encuentran incrementos periódicos en las medidas correctivas por consumo de bebidas con alcohol, especialmente en los días sábado y domingo, en consonancia con ese consumo social de fines de semana que se ha descrito para Colombia (Pérez-Gómez et al., 2018) y en otros contextos, mientras que en cuanto a los horarios, los CCC son detectados en casi todos los momentos del día, incluyendo la noche y la madrugada, pero especialmente en los horarios de actividades laborales y comerciales: primeras horas de la mañana y entre las 4: 00 y los 8:00 pm, en correspondencia con la necesidad de comprar alimentos, bebidas y otros bienes y servicios de primera necesidad, según los horarios respectivos, unido a la venta de bebidas alcohólicas en horarios nocturnos. Así, la comisión de ciertas conductas contrarias a la convivencia se acomoda también a los postulados de la teoría de la rutina de actividades (Andresen \& Malleson, 20I5; Bernie \& Messerschmidt, 2006).

En tercer lugar, no se puede dejar de lado los resultados hallados entre la edad y la comisión de CCC. Estos se concentran en las edades más jóvenes, entre los 18 y los 24 años y, a continuación, entre los 25 y los 30 años. Queda para investigaciones futuras indagar sobre dicha asociación y sus causas. Estas pueden ser diversas, desde aspectos de posibles sesgos de los funcionarios policiales a la hora de detectar los CCC y sancionar a jóvenes (Altell, 22 de mayo de 2020), pasando por la mayor toma de riesgos en adolescentes y jóvenes, tal como se encuentra con relación a los juegos de azar (Chan-Gamboa, Ruiz-Pérez, Morales-Quintero \& Echeburúa 2019), los accidentes viales o el consumo de drogas, o factores socioeconómicos que subyacen a esa toma de riesgo, como, por ejemplo, el desempleo, la inseguridad alimentaria u otras (Klaitman, Solomonov, Yaloz \& Biswas, 2018).

Por otra parte, es necesario reconocer con relación a las infracciones, las faltas o los delitos menores la diversidad de fuentes de información para su registro y la probable existencia de una cifra oscura, tal y como se postula para 
la criminalidad convencional (Ruiz-Pérez, 20I4a; Tamayo \& Norza, 2018). Reducir dicha cifra negra y esclarecer los determinantes de cada tipo de medidas correctivas puede contribuir a establecer actos administrativos y normas legales de mayor conducencia, así como políticas de convivencia ciudadana más eficaces, las cuales pueden tener implicaciones a su vez en ámbitos diferentes de la justicia, como, por ejemplo, la salud pública. Esto incluye la identificación y el estudio de los factores explicativos de las tasas de CCC. La extensión y el objetivo principal del presente trabajo hace que el papel de variables como las económicas (e.g., tipos de actividad económica de la región, índice de Gini, tasa de población por debajo del umbral de la pobreza, entre otras), de tejido social (eficacia colectiva, cultura ciudadana, resiliencia comunitaria e.g.), o de criminalidad convencional, deban abordarse en estudios posteriores. Con todo, de mantenerse las medidas de confinamiento por el resto del 2020, los registros administrativos de CCC por estas faltas podría superar el millón de registros en Colombia, lo que significa un desacatamiento general de los diferentes actos administrativos, independiente de las motivaciones internas $\circ$ externas de los infractores.

\section{Agradecimientos}

A la Policía Nacional de Colombia, en particular al Grupo de Registro Nacional de Medidas Correctivas de la Dirección de Seguridad Ciudadana y al Grupo de Información Criminal de la Dirección de Investigación Criminal e Interpol, por la información de registros administrativos suministrados. A los revisores, por ayudar a mejorar sustancialmente aspectos formales del manuscrito.

\section{Referencias}

Andresen, M.A., \& Malleson, N. (20I5). Intra-week spatial-temporal patterns of crime. Crime Science, 4(I2), I-I I. https:// doi.org/10.1 186/s40163-015-0024-7

Aksoy, E. (2017). Geography of crime and its relation to location: the city of Balikesir (Turkey). IOP Conference Series: Materials Science and Engineering, 245, I-II. https://doi. org//0.1088/I757-899X/245/7/072012

Altell, G. (22 de mayo de 2020). Distanciamiento social o el proceso de individualización. ElPeriódico.com. https://www. elperiodico.com/es/opinion/20200522/articulo-gemmaaltell-distanciamiento-social-o-el-proceso-de-individualizacion-7971777
Arenas, G. (2I de mayo de 2020). Erradicación y protestas sociales en Putumayo, una bomba de tiempo. https://miputumayo. com.co/2020/02/29/erradicacion-y-protestas-sociales-enputumayo-una-bomba-de-tiempo/

Ashby, M. P.J., \& Bowers, K.J. (20I3).A comparison of methods for temporal analysis of aoristic crime. Crime Science, 2(1), I- | 6. https://doi.org/ | 0. I | 86/2193-7680-2- I

Ávila-Jiménez, C.(2I de mayo de 2019).Así es la vida en Sáchica, el pueblo que más bebe cerveza en Colombia.ElTiempo.https:// www.eltiempo.com/colombia/otras-ciudades/sachica-elpueblo-colombiano-que-mas-toma-cerveza-86312

Beirne, P., \& Messerschmidt, J.W. (2006). Criminology. Roxbury Publishing Company.

Caracol Radio Tunja. (I I de julio de 2020). 'Enguayabados' en Boyacá por IVA a la cerveza. https://caracol.com.co/emisora/20 I8/I2/06/tunja/I544 I |4 I35_46747I.html.

Caracol Radio Barranquilla. (2I de mayo de 2020). Soledad evalúa militarizar sus calles por incumplimiento de la cuarentena. https:/caracol.com.co/emisora/2020/04/30/ barranquilla/I588254II9_4I5853.html

Chan-Gamboa, E. C., Ruiz-Pérez, J. I., Morales-Quintero, L.A., \& Echeburúa, E. (2019). Creencias sobre los juegos de azar y síntomas de juego patológico: propiedades psicométricas y prevalencia en estudiantes mexicanos. Revista Mexicana de Psicología, 36(2), |47-I 59.

Departamento Administrativo Nacional de Estadística. (23 de mayo de 2020). Proyecciones de población. https://www. Dane.Gov.Co/Index.Php/Estadisticas-Por-Tema/Demografia-Y-Poblacion/Proyecciones-De-Poblacion

EI Tiempo. (4 de julio de 2020a). Indisciplinados hacen "conejo" a comparendos por violar la cuarentena. https://www.eltiempo.com/colombia/otras-ciudades/cuarentena-en-colombia-comparendos-por-violar-cuarentena-no-son-cancelados- 497326

El Tiempo. (24 de abril de 2020). El indignante "top" de frestas en plena cuarentena nacional. https://www.eltiempo.com/justicia/investigacion/coronavirus-en-colombia-fiestas-durantela-cuarentena-nacional-486916

Foronda,V. de. (I80 I). Cartas sobre la policía. Imprenta de Cano.

Herrera, H. (I7 de abril de 2020). En un 93\% se cumple confinamiento en Barranquilla y su área metropolitana. El Heraldo. https://www.elheraldo.co/judicial/en-un-93se-cumple-confinamiento-en-barranquilla-y-su-areametropolitana-7|863|

Jendryke, M. \& McClure, S.C. (2019). Mapping crime - Hate crimes and hate groups in the USA: A spatial análisis with gridded data. Applied Geography, III. https://doi. org/10.1016/j.apgeog.2019.102072 
Justi, J. C. von. (1996). Ciencia del Estado. Instituto de Administración Pública del Estado de México.

Klaitman, S. S., Solomonov, E., Yaloz A., \& Biswas, S. (2018). The incidence of road traffic crashes among young people aged 15-20 years: differences in behavior, lifestyle and sociodemographic indices in the Galilee and the Golan. Frontiers in Public Health, 6, 202. https://doi.org//0.3389/ fpubh.2018.00202

Lamba, I. (2020). Why India needs to extend the nationwide lockdown. American Journal of Emergency Medicine, 38, 1528-1599. https://doi.org/10.1016/j.ajem.2020.04.026

Maguire, M., Morgan, R., \& Reiner, R. (2002). Manual de Criminología. Oxford

Muñoz-Sabaté, LI. (1980). Métodos y elementos para una psicología jurídica. En L. L. Muñoz-Sabaté, F. Munnem, \& R. Bayes (Eds.), Introducción a la psicología jurídica (pp. 15-46). Trillas.

Paital, B., Das, K., \& Kumar, S. (2020). Internation social lockdown versus medical care against COVID-19, a mild environment insight with special reference to India. Science of Total Environment, 728, 1-17. https://doi.org/10.1016/j. scitotenv.2020.1389/4

Parmet, W. E., \& Sinha, M. S. (2020). COVID-19-The law and limits of quarantine. The New England Journal of Medicine, 382(I5), e28(I)-e28(3). https://doi.org/I0.1056/NEJMp20042II

Pérez-Gómez, A., Lanziano, C., Reyes-Rodríguez, M.F., MejíaTrujillo, J., \& Cardozo-Macías, F. (2018). Perfiles asociados al consumo de alcohol en adolescentes colombianos. Acta Colombiana de Psicología, 2 I (2), 258-269. https://doi. org/I0.147I8/ACP.2018.2I.2.12

Revista Semana. (2I de mayo de 2020). La voz de protesta de los indígenas amazónicos ante manejo de la pandemia. https://sostenibilidad.semana.com/impacto/articulo/coronavirus-en-colombia-la-voz-de-protesta-de-los-indigenasamazonicos-ante-manejo-de-la-pandemia/50507

Revista Semana. (s. f). 10 municipios colombianos y 10 países donde más se consume cerveza. https://especiales.semana. com/especiales/los-10-mas/asi-somos/ / 0-municipioscolombianos-10-paises-donde-mas-consumen-cerveza.html
Ruiz, J. I. (20|4a). Cuestiones en criminología comparada y en percepción de seguridad. En J. I. Ruiz (Ed.), Psicología Social y Justicia (pp. 36I-397). Universidad Nacional de Colombia, Colegio Colombiano de Psicólogos.

Ruiz, J. I. (20/4b). Sistemas de información geográfica e indicadores psicosociales: el caso del miedo al crimen. En J. I. Ruiz (Ed.), Psicología Social y Justicia (pp. 339-360). Universidad Nacional de Colombia, Colpsic.

Salje, H., Tran-Kiem, C., Lefrancq, N., Courtejoie, N., Bosetti, P., Paireau, J., ..., \& Cauchemez, S. (2020). Estimating the burden of SARS-CoV-2 in France. Science, 6500, 208-21I. https://doi.org/10.1 I26/science.abc35 I7

Tamayo, F. L., \& Norza, E. (2018). Midiendo el crimen: cifras de criminalidad y operatividad policial en Colombia, año 2017. Revista Criminalidad, 60(3), 49-7I.

Torres-Rico, R. (2008). Delitos y contravenciones como factores de criminalidad y de perturbación de la convivencia social. Revista Criminalidad, 50(I), 85-98.

Tramahullas, G. (I I de abril de 2020). La salud pública como motor de la historia. El Periódico. https://www.elperiodico. com/es/cuaderno/202004I I/cambios-sociales-a-la-luz-delas-epidemias-historicas-792/757

\section{Referencias legales}

Policía Nacional. (2020a). Registro Nacional de Medidas Correctivas. Dirección de Seguridad Ciudadana.

Policía Nacional. (2020b). Circular 007. Dirección GeneralOficina de Planeación.

Policía Nacional. (2020c). Delitos de impacto social. Dirección de Investigación Criminal e Interpol.

República de Colombia. (2016). Ley I80I de 29 de julio. Por medio de la cual se expide el Código Nacional de Policía y Convivencia. Congreso de la República.

República de Colombia. (2019). Ley 2000 de 14 de noviembre. Por medio de la cual se modifica el Código Nacional de Policía y Convivencia. Congreso de la República. 